\title{
Thermally Induced Nanocrystal Array of Poly(N-Vinylcarbazole) on Si-Wafer Substrate
}

\author{
Kazushi Yamada ${ }^{*}$, Yasuyuki Tsuboi ${ }^{2}$ \\ ${ }^{1}$ Advanced Fibro-Science, Kyoto Institute of Technology, Kyoto, Japan \\ ${ }^{2}$ School of Science, Osaka City University, Osaka, Japan \\ Email: ${ }^{*}$ kazushi@kit.ac.jp
}

Received 21 January 2014; revised 26 February 2014; accepted 19 March 2014

Copyright (C) 2014 by authors and Scientific Research Publishing Inc.

This work is licensed under the Creative Commons Attribution International License (CC BY). http://creativecommons.org/licenses/by/4.0/

(c) (i) Open Access

\begin{abstract}
Recently, nanostructures such as nanocrystals and nanoaggregates have attracted much attention in many quarters of materials, electronics, and biology to create higher-value-added functional nanoscale materials and films. In this research, the fabrication of nanoaggregates on ultrathin photoconductive films of poly( $\mathrm{N}$-vinylcarbazole) (PVCz) by applying thermal treatment is demonstrated. The structure and size are discussed on the basis of the results of atomic force microscope images. As a result, after thermal treatment of these films above the glass transition temperature $\left(T_{g}\right)$ of $\mathrm{PVCz}$, different types of surface morphological changes were induced showing a dependence on the tacticity of PVCz. Radically polymerized PVCz(r) ultrathin film showed small aggregates with heights of $\sim 8 \mathrm{~nm}$ on the film surface after thermal treatment, while cationically polymerized PVCz(c), which has higher isotactic diad fractions than $\mathrm{PVCz}(\mathrm{r})$, indicated similar aggregates on the film surface, although the number of aggregates was smaller than $\mathrm{PVCz}(\mathrm{r})$. It is considered that these different phenomena depend on the tacticity of PVCz and the interaction between PVCz molecules and the substrate surface.
\end{abstract}

\section{Keywords}

AFM, Poly(N-Vinylcarbazole), Structural Transfer, Thermal Treatment, Nanoaggregates

\section{Introduction}

Recently, nanostructures such as nanocrystals and nanoaggregates have attracted much attention in many quar-

"Corresponding author. 
ters of materials, electronics, and biology to create the high-value-added functional nanoscale materials and films. Our research groups have also investigated the fabrication of functional films with nanostructures prepared by applying the selective doping method of photoactive chromophore [1] [2] and the laser ablation technique for gold nanoparticles/polymer films [3] [4]. However, photoconductive polymers have attracted increasing attention for producing nanodevices, such as optical sensors, photovoltaic cells, and so on. Poly(N-vinylcarbazole) (PVCz) is a photoconductive polymer, and it has received much attention as a representative photoconductive aromatic vinyl polymer. In addition, systematic studies have been carried out extensively covering photocurrent, luminescence, and absorption measurements in addition to structural studies in order to elucidate its mechanical, physical, chemical, and electronic properties [5] [6].

Concerning the structural studies, it was revealed that the tacticity of $\mathrm{PVCz}$ depends on the polymerization method (radical (r) or cationic (c) polymerization) on the basis of the nuclear magnetic resonance (NMR) spectra and glass transition temperatures $\left(T_{g}\right)$ of $\mathrm{PVCz}$ [7]. Furthermore, other research groups had investigated the relation between the photophysical properties of PVCz and its tacticity, and the difference in tacticity was reflected in the drift mobility and in the thermal crystallization behavior [8] [9]. However, the thermal crystallization of PVCz(c) films is much more difficult than that of $\mathrm{PVCz}(\mathrm{r})$ films, and its mobility hardly changes with thermal treatment. In addition, it has been reported that $\mathrm{PVCz}$ (c) shows three different values of $T_{g}$, while PVCz(r) shows only one [10]-[12]. The triple $T_{g}$ of the former was interpreted by the correspondence to the whole chain and the syndiotactic and isotactic stereoblocks, while the single $T_{g}$ of the latter was interpreted by an absence of long sequences that behave as separate phases due to stereoblocks. That is, the length of each sequence in the stereoblock structure of $\mathrm{PVCz}(\mathrm{r})$ is considered to be too short to behave as a separate phase for $T_{g}$. However, these investigations focused on bulk samples such as thick film, bulk, or powder samples in order to measure the NMR, X-ray diffraction, and so on, and not on nanoscale samples such as ultrathin films on substrate. Therefore, it is very important to understand ultrathin PVCz films on a solid substrate at the nanoscale.

In this research, the thin films and ultrathin films of PVCz on Si-wafer substrate were prepared by the cast method, and these films were thermally treated in order to induce the crystallization of PVCz. The surface morphology changes of the PVCz films are discussed on the basis of the results of atomic force microscopy (AFM) observation.

\section{Experimental}

\subsection{Synthesis of PVCz}

$N$-vinylcarbazole (VCz) was purified by the usual method from commercial product. $\mathrm{PVCz}(\mathrm{r})$ was prepared by polymerization of a 1.0-M benzene solution of the purified $\mathrm{VCz}$ in the presence of azobisisobutyronitrile $(0.4$ $\mathrm{mol} \%$ ) in an evacuated sealed tube at $60^{\circ} \mathrm{C}$ for $9 \mathrm{~h}$. $\mathrm{PVCz}(\mathrm{c})$ was prepared by polymerization of a $0.3-\mathrm{M}$ toluene solution of purified $\mathrm{VCz}$ in the presence of $1 \mathrm{~mol} \%$ boron trifluoride-diethyl ether (BF3.OEt2) under a nitrogen atmosphere at room temperature for $1.5 \mathrm{~h}$. The $\mathrm{PVCz}$ samples were purified by reprecipitation 3 times from benzene with methanol. The chemical structure of PVCz is shown in Figure 1.

\subsection{Sample Preparation}

PVCz films were cast on silicon (Si) wafers of approximately $10 \times 10 \mathrm{~mm}^{2}$ from a 1,2-dichloroethane solution at room temperature and dried in vacuo for several hours. The average thickness of the amorphous PVCz film was estimated from the weight and density of a film with known area. Thermal treatment of PVCz films on Si wafer substrate was carried out at $583 \mathrm{~K}$ above the $T_{g}$ under high vacuum for $1 \mathrm{~h}$.

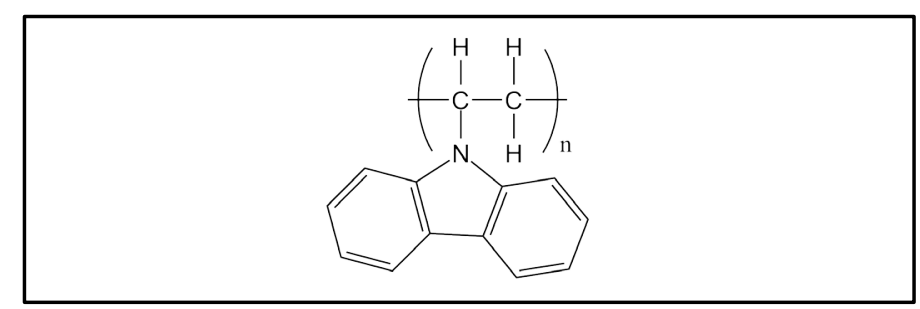

Figure 1. Chemical structure of poly(N-vinylcarbazole) (PVCz). 


\subsection{DSC Measurement and WAXD Measurement}

Differential scanning calorimetry (DSC; DSC2920, TA Instruments, New Castle, Delaware) was conducted in a nitrogen atmosphere at a temperature range of $50-350^{\circ} \mathrm{C}$ with a heating rate of $20^{\circ} \mathrm{C} / \mathrm{min}$ for the purpose of investigating the thermal properties of the PVCz films. Wide-angle X-ray diffraction (WAXD) patterns were taken on an X'Pert-Pro MPD X-ray diffractometer for PVCz(r) and PVCz(c) samples.

\subsection{AFM Observation}

An atomic force microscope (Seiko Instruments, Inc., SPI 3800N/SPA-400 system) was used in dynamic force mode under ambient conditions. Si single crystal tips with a cantilever length of $125 \mu \mathrm{m}$ were used. Both height and phase images were recorded simultaneously. Since the soft amorphous material is more compliant than the hard crystalline material, a phase difference between the two materials provides a distinct contrast between these states in the phase image.

\section{Results and Discussion}

\subsection{Thermal Properties of PVCz with DSC and WAXD Measurement}

DSC and WAXD measurement were performed for PVCz(r) and $\mathrm{PVCz}(\mathrm{c})$ powder. The results of DSC and WAXD of PVCz(r) and PVCz(c) are shown in Table 1. For the DSC measurement, the base line shift which was corresponding to glass transition temperature $\left(T_{g}\right)$ were observed at $502 \mathrm{~K}$ and $518 \mathrm{~K}$, respectively. Accordingly, thermal treatment of PVCz films on Si wafer substrate was carried out at $583 \mathrm{~K}$ above the $T_{g}$ in this investigation. On the other hand, heating of PVCz films to a temperature above $T_{g}$ under high vacuum resulted in the appearance of a narrow crystalline X-ray scattering peak at $2 \theta=8.39^{\circ}$, which indicates the occurrence of paracrystalline lamellar [9]. The value of the peak width at half height (FWHM) $(\Delta 2 \theta)$ of PVCz(r) was smaller than that of $\mathrm{PVCz}(\mathrm{c})$. Therefore, it is considered that the significant difference between the thermally treated $\mathrm{PVCz}(\mathrm{r})$ and PVCz(c) samples in the results of DSC and WAXD is attributed to the difference in their thermal crystallization behavior.

\subsection{AFM Observation of Thin PVCz Films with a Thickness of $\sim 10 \mu \mathrm{m}$}

AFM images of $\mathrm{PVCz}(\mathrm{r})$ and $\mathrm{PVCz}(\mathrm{c})$ films cast on Si wafer substrate before and after thermal treatment shown in Figure 2. The scales of the height images are $10 \mathrm{~nm}$ in Figures 2(a)-(c) and $150 \mathrm{~nm}$ in Figure 2(d). The surface of both the as-cast films was smooth, and no difference between PVCz(r) and PVCz(c) films in the surface morphology was found. However, a large difference between thermally treated PVCz(r) and PVCz(c) films was observed: the AFM image of the thermally treated PVCz(r) films showed a vein-like structure and large lumps with a difference in height of more than $100 \mathrm{~nm}$, while no such structure and lumps were observed for the thermally treated $\mathrm{PVCz}$ (c) films. That is, thermal treatment at a temperature above $T_{g}$ under high vacuum induced a drastic change in the surface morphology of cast $\mathrm{PVCz}(\mathrm{r})$ films, whereas the surface morphological change of $\mathrm{PVCz}(\mathrm{c})$ films was slight. These results indicate that the thermal treatment of thin PVCz films under high vacuum results in tacticity-dependent change in the surface morphology of $\mathrm{PVCz}$ of a homopolymer.

Under a polarizing microscope, a bright image was observed for the thermally treated PVCz(r) films. For the thermally treated PVCz(c) films, however, a dark image was observed, and growth of spherulite morphology was not observed. These tacticity-dependent results were interpreted as the thermal crystallization of $\mathrm{PVCz}(\mathrm{r})$ films being much easier than that of $\mathrm{PVCz}$ (c) films. Hence, the significant difference between the thermally treated PVCz(r) and PVCz(c) films in the AFM images is attributed to the difference in their thermal crystallization behavior. Figure 2 shows the tacticity-dependent thermal crystallization behavior of a homopolymer as a

Table 1. Results of DSC and WAXD measurement for PVCz samples.

\begin{tabular}{cccc} 
& Tg (K) & 2o & FWHM \\
\hline $\operatorname{PVCz}(\mathrm{r})$ & 502 & $8.39^{\circ}$ & $0.13^{\circ}$ \\
$\operatorname{PVCz}(\mathrm{c})$ & 518 & $8.39^{\circ}$ & $0.30^{\circ}$ \\
\hline
\end{tabular}

"FWHM = "full width at half maximum". 


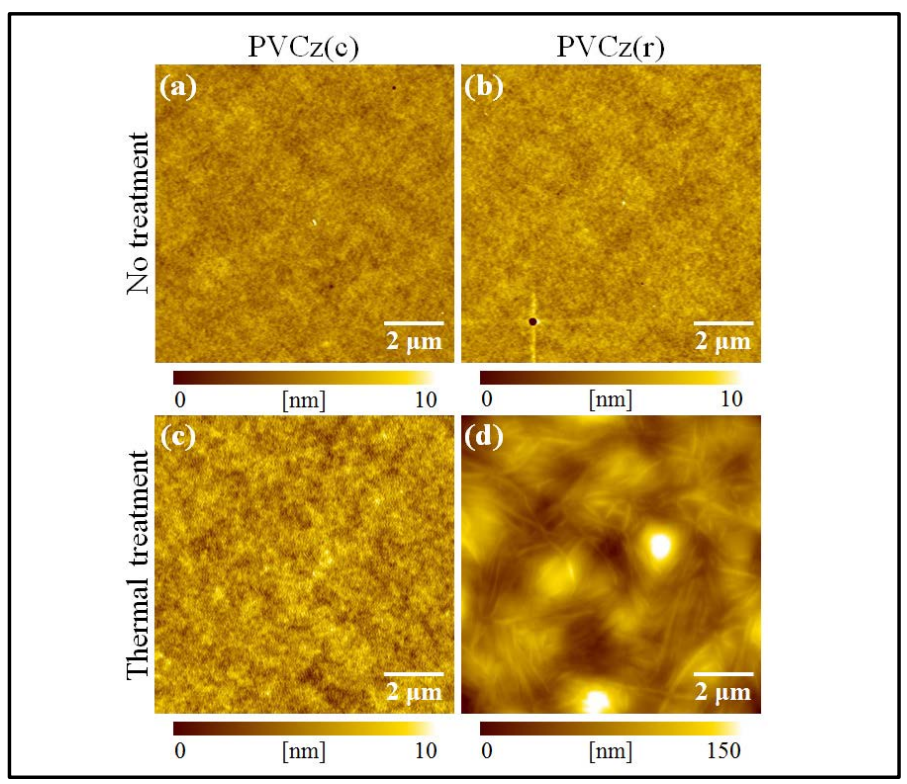

Figure 2. AFM images of thin PVCz films with $10 \mu \mathrm{m}$ thickness cast on silicon wafer substrate before and after thermal treatment at $583 \mathrm{~K}$ under high vacuum for $1 \mathrm{~h}$.

surface-morphological change in AFM images.

As expected from the above results, the AFM phase images of the thermally treated $\mathrm{PVCz}(\mathrm{r})$ and $\mathrm{PVCz}(\mathrm{c})$ films were quite different: no contrast was found in the phase image of the $\mathrm{PVCz}(\mathrm{c})$ films, while the phase image of the $\mathrm{PVCz}(\mathrm{r})$ films exhibited distinct contrast revealing a veined surface structure.

\subsection{AFM Observation of Ultrathin PVCz Films with a Thickness of $~ 10 \mathrm{~nm}$}

The ultrathin films the $10 \mathrm{~nm}$ thickness cast from very dilute PVCz solutions was prepared on Si wafer in order to investigate the morphological changes with thermal treatment above the Tg of PVCz. These films were observed by AFM, and the results are shown in Figure 3. As shown in this figure, the ultrathin PVCz film before thermal treatment has a flat surface; however, the surface shape was drastically changed by thermal treatment, i.e., small aggregated islands with unique shapes appeared on the ultrathin film surface. The number of unique aggregates for ultrathin $\mathrm{PVCz}(\mathrm{r})$ film was larger than for ultrathin $\mathrm{PVCz}(\mathrm{c})$ film. It is considered that the $\mathrm{PVCz}(\mathrm{r})$ allows the growth of crystals on the ultrathin films under thermal treatment as compared with $\mathrm{PVCz}(\mathrm{c})$, and this phenomenon is consistent with the result of the dependence of tacticity for PVCz molecules as shown in Figure 2.

Figure 4 shows magnified height and phase images, a lateral image, and an aggregate image of ultrathin PVCz(r) film. From the phase image in Figure 4(a), the small aggregated islands correspond to a crystal structure with a low phase shift as compared with the flat surface area, because the hard part, which corresponds to the crystal area, indicates a weaker interaction between the Si-tip of AFM and the ultrathin film surface as compared with the soft part of the amorphous area. In addition, these small aggregated islands have a unique shape and size with $\sim 8 \mathrm{~nm}$ height as shown in Figure 4(b).

Figure 5 shows graphs of frequency distributions for the unique aggregates, and these distributions correspond to Gaussian distributions. The peak value of crystal width is $\sim 200 \mathrm{~nm}$, and the peak value of crystal length is $\sim 400 \mathrm{~nm}$. Therefore, it is considered that these nanoaggregates grew from crystal nucleations on the amorphous film of PVCz after thermal treatment. In addition, we show in the schematic illustrations of the crystal growth process of PVCz that the nanocrystals consist of small lamella structures on the ultrathin PVCz film as shown in Figure 6.

In this investigation, we prepared the ultrathin PVCz films with $15 \mathrm{~nm}$ thickness on Si-wafer substrate. It is noted that the average thickness of the flat area is most probably less than twice the radius of gyration of the PVCz molecules in the neat films [13], although the radius of gyration of the PVCz(r) and PVCz(c) molecules in 


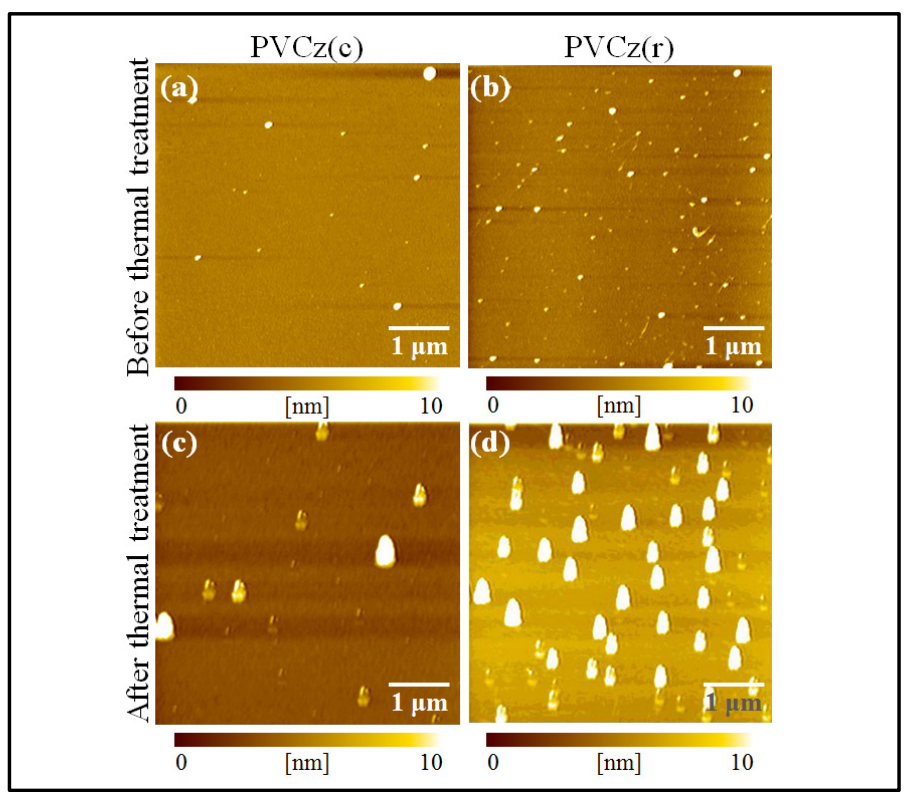

Figure 3. AFM images of ultrathin PVCz films with $10 \mathrm{~nm}$ thickness cast on silicon wafer substrate before and after thermal treatment at $583 \mathrm{~K}$ under high vacuum for $1 \mathrm{~h}$.

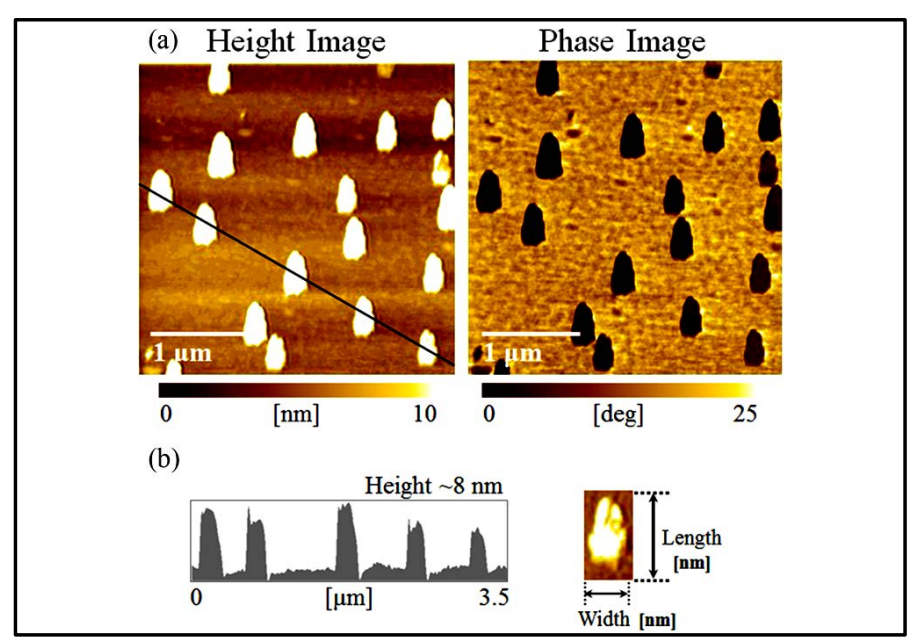

Figure 4. Typical AFM images of ultrathin PVCz(r) film after thermal treatment at $583 \mathrm{~K}$ under high vacuum for $1 \mathrm{~h}$, (a) height and phase image and (b) lateral image of PVCznanocrystals.

the neat films is generally unknown. However, we succeeded in fabricating a nanocrystal array of PVCz on ultrathin PVCz films by applying thermal treatment above the $T_{g}$ under high vacuum condition. In addition, the dependence is on the tacticity of PVCz. That is, the ultrathin PVCz(r) film prepared by radical polymerization showed small aggregates with a height of $\sim 8 \mathrm{~nm}$ on the film surface after thermal treatment, while cationically polymerized $\mathrm{PVCz}(\mathrm{c})$, which has higher isotactic diad fractions than $\mathrm{PVCz}(\mathrm{r})$ also indicated the same aggregates on the film surface, although the number of aggregates is lower than that on $\mathrm{PVCz}(\mathrm{r})$. Therefore, it is considered that these different phenomena depend on the tacticity of PVCz and on the interaction between PVCz molecules and the substrate surface.

\section{Conclusion}

In this research, we demonstrated a thermally induced nanocrystal array of poly(N-vinylcarbazole) on Si-wafer 

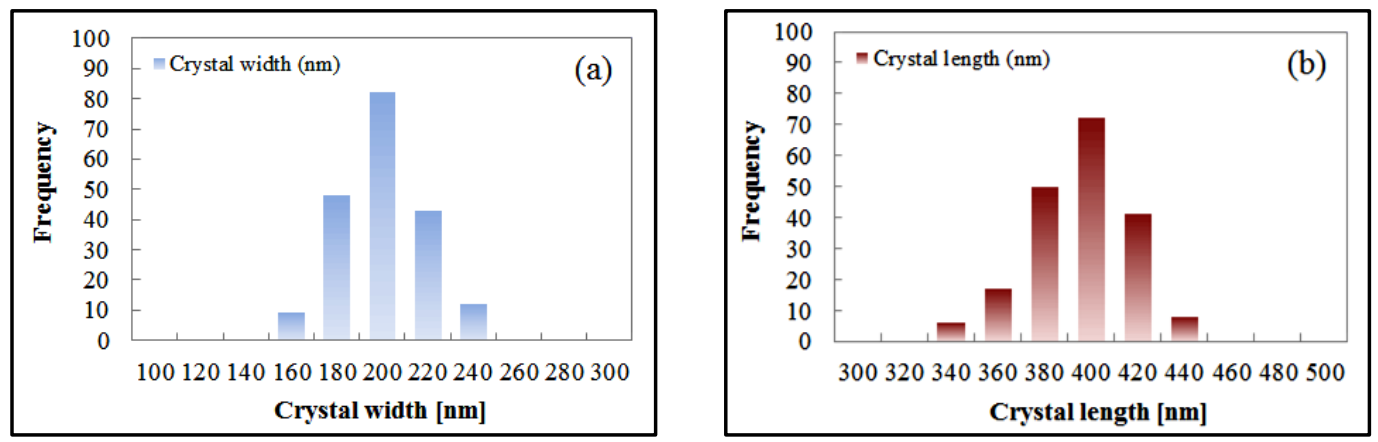

Figure 5. Histograms of PVCznanocrystal size: (a) width [nm] and (b) length [nm].

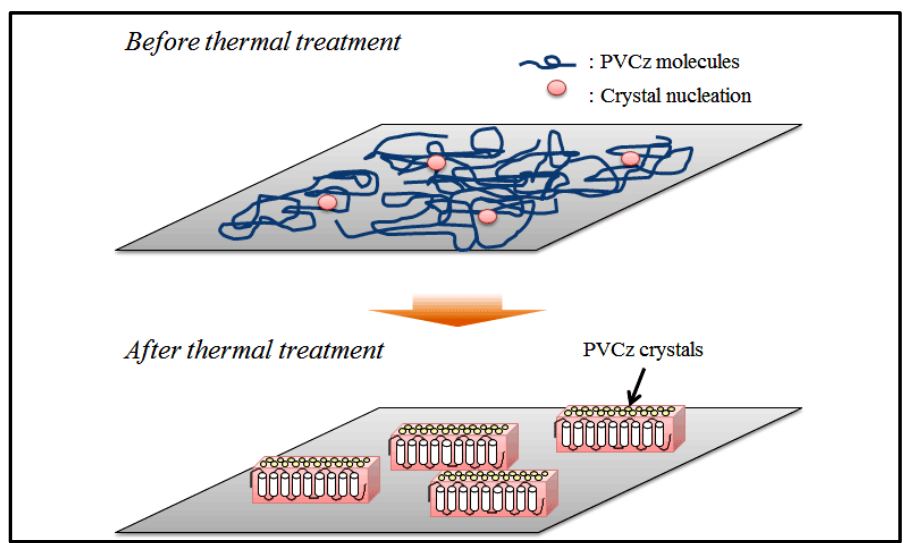

Figure 6. Schematic illustration of the crystal growth process of $\mathrm{PVCz}$ nanocrystal on the ultrathin PVCz films.

substrate. The thin films and ultrathin films of PVCz on Si-wafer substrate were prepared by a cast method, and these films were thermally treated in order to induce the crystallization of PVCz. For PVCz film with $\sim 10 \mu \mathrm{m}$ thickness, it was found that thermal treatment above $T_{g}$ under high vacuum induces a drastic change in the surface morphology of cast $\mathrm{PVCz}(\mathrm{r})$ films, whereas the surface morphological change of $\mathrm{PVCz}(\mathrm{c})$ films is slight. These results indicate that thermal treatment of thin PVCz films under high vacuum causes tacticity-dependent change in the surface morphology of PVCz of a homopolymer. However, for an ultrathin PVCz film with 15 $\mathrm{nm}$ thickness, we succeeded in fabricating a nanocrystal array of $\mathrm{PVCz}$ on ultrathin $\mathrm{PVCz}$ films by applying thermal treatment above $T_{g}$ under high vacuum. In addition, the dependence is on the tacticity of PVCz. The present results suggest that both the AFM images of ultrathin PVCz films and the surface morphological change induced by thermal treatment are very sensitive to the substrate surface property. In order to elucidate further the origin of these phenomena and to control the arrangement of small $\mathrm{PVCz}$ crystal aggregates, detailed studies of the surface-property dependence will be carried out in near future.

\section{Acknowledgements}

The authors thank honorary professor Akira Itaya of the Kyoto Institute of Technology in Japan for technical contribution and polymer synthesis.

\section{References}

[1] Machida, S., Nakata, H., Yamada, K. and Itaya, A. (2007) Morphological Change of a Diblock Copolymer Film Induced by Selective Doping of a Photoactive Chromophore. Journal of Polymer Science: Part B: Polymer Physics, 45, 368-375. http://dx.doi.org/10.1002/polb.21050

[2] Machida, S., Nakata, H., Yamada, K. and Itaya, A. (2006) Position-Selective Arrangement of Nanosized Polymer Microsphere on Diblock Copolymer Film with Sea-Island Microphase Structure. Japanese Journal of Applied Physics, 45, 4270-4273. http://dx.doi.org/10.1143/JJAP.45.4270 
[3] Yamada, K., Itoh, T. and Tsuboi, Y. (2008) Nanohole Processing of Polymer Films Based on the Laser-Induced Superheating of Au Nanoparticles. Applied Physics Express, 1, 087001. http://dx.doi.org/10.1143/APEX.1.087001

[4] Muraoka, K., Yamada, K., Shoji, T., Sugimura, H., Kitamura, N. and Tsuboi, Y. (2012) Nano Porous Films Processing of Polymer Films Based on Localized Surface Plasmon Resonance of Au Nanoparticles. Journal of Laser Micro/Nanoengineering, 7, 260-263.

[5] Penwell, R.C., Ganguly, B.N. and Smith, T.W. (1978) Poly(N-Vinylcarbazole): A Selective Review of Its Polymerization, Structure, Properties, and Electrical Characteristics. Journal of Polymer Science: Macromolecular Reviews, 13, 63-160. http://dx.doi.org/10.1002/pol.1978.230130102

[6] Peason, J.M. and Stolka, M. (1981) Poly(N-Vinylcarbazole). Gordon and Breach Science Publishers, New York.

[7] Okamoto, K., Yamada, M., Itaya, A., Kimura, T. and Kusabayashi, S. (1976) Polymerization of N-Vinylcarbazole, N-Vinyl-5H-benzo[b]Carbazole, and N-Vinyl-7H-Benzo[c]Carbazole. Macromolecules, 9, 645-649. http://dx.doi.org/10.1021/ma60052a023

[8] Itaya, A., Okamoto, K. and Kusabayashi, S. (1976) Emission Spectra of the Vinyl Polymers with Pendant Carbazolyl Groups. Bulletin of the Chemical Society of Japan, 49, 2082-288. http://dx.doi.org/10.1246/bcsj.49.2082

[9] Itaya, A., Okamoto, K. and Kusabayashi, S. (1985) Hole Transport in Amorphous Films of Poly(N-Vinylcarbazole) Copolymers of N-Vinylcarbazole with STYRENE, Polystyrene Molecularly-Doped with N-Isopropylcarbazole, and 1,3-Di(N-carbazolyl)Propane. Polymer Journal, 17, 557-565. http://dx.doi.org/10.1295/polymj.17.557

[10] Terrel, D.R. and Evers F. (1982) Stereocontrol in the Cationic Polymerization of N-Vinylcarbazole. Journal of Polymer Science Part A: Polymer Chemistry, 20, 2529-2543. http://dx.doi.org/10.1002/pol.1982.170200913

[11] Terrel, D.R. and Evers, F. (1982) Stereocontrol in the Free Radical Polymerization of N-Vinylcarbazole. Macromolecular Chemistry and Physics, 183, 863-874.

[12] Terrel, D.R., Evers, F., Smoorenburg, H. and van den Boaert, H.M. (1982) The Dependence of the Glass Transition Temperature of Poly(N-Vinylcarbazole) upon Steric Microstructure and Molecular Weight. Journal of Polymer Science: Polymer Physics Edition, 20, 1933-1945. http://dx.doi.org/10.1002/pol.1982.180201016

[13] Leon, L.M., Katime, I. and Rodroguez, M. (1979) Solution Properties of Poly(N-vinyl carbazole)—II. Thermodynamic Properties in Various Solvent. European Polymer Journal, 15, 29-34. (For Example, the Radius of Gyration of PVCz(r) in 1,2-Dichloroethane, Casting Solvent, Is Estimated to Be $26 \mathrm{~nm}$ for Mw = 360000). 